\title{
Nuevas tecnologías y aprendizaje significativo de las ciencias
}

\section{ICT and meaningful science learning}

\author{
Marta Romero Ariza \\ mromero@ujaen.es \\ Antonio Quesada Armenteros \\ antquesa@ujaen.es \\ Departamento de Didáctica de las Ciencias \\ Universidad de Jaén
}

RESUMEN • Este trabajo pretende fomentar la reflexión sobre el potencial de la tecnología educativa para promover el aprendizaje significativo de las ciencias. Con este propósito se recurre a la literatura especializada, para mostrar algunos resultados de investigación sobre la aplicación de las TIC a la enseñanza de las ciencias (herramientas de adquisición de datos, programas de modelización, simulaciones, laboratorios virtuales...). El valor formativo de dichas aplicaciones se discute desde los actuales conocimientos acerca de cómo los individuos aprenden, mostrando el potencial de estos recursos para superar los obstáculos específicos asociados al aprendizaje efectivo de las ciencias (ideas previas, falta de contextos significativos, grado de abstracción de modelos y teorías...). No obstante, también se ofrece una visión crítica señalando riesgos y limitaciones.

PALABRAS CLAVE: TIC; enseñanza de las ciencias; obstáculos específicos; laboratorios virtuales; simulaciones; herramientas de adquisición de datos; trabajo colaborativo apoyado en ordenador.

ABSTRACT - This paper intends to promote reflection on ICT use to facilitate meaningful science learning. In order to achieve this goal, key research works on technology implementation (data acquisition devices, modeling tools, simulations, virtual laboratories...) are selected from the specialized literature. The instructional value of these applications is studied from the current knowledge on how we learn. The analysis shows the potential of technology to overcome common science learning troubles (students' previous ideas, lack of meaningful contexts, abstract scientific models and theories...). A critical view is also shown, pointing out risks and limitations associated with ICT implementation for science education.

KEYWORDS: educational technology; science education; science learning difficulties; virtual laboratories; simulations; data acquisition; CBCL. 


\section{INTRODUCCIÓN Y OBJETIVOS}

El objetivo de este trabajo es analizar la influencia de algunas aplicaciones tecnológicas en la enseñanza de las ciencias experimentales, así como invitar a la reflexión sobre el uso de la tecnología y sus posibles efectos en la calidad del aprendizaje adquirido por los estudiantes.

Para ello, se ha llevado a cabo una revisión de la literatura especializada y se han descrito algunos proyectos e investigaciones, basadas en la integración de la tecnología en los procesos de enseñanzaaprendizaje. Sin embargo, es necesario aclarar que el principal propósito de este artículo no es ofrecer una revisión minuciosa de todos los trabajos relacionados con la aplicación de las TIC a la enseñanza de las ciencias, sino más bien seleccionar algunos ejemplos que pueden resultar especialmente ilustrativos para promover la reflexión en torno a la influencia de la tecnología en el aprendizaje de las ciencias.

El análisis del impacto de las TIC sobre la enseńanza de las ciencias se realiza en todo momento desde los actuales conocimientos acerca de cómo los individuos aprenden. En este sentido, se presta especial atención a los obstáculos específicos asociados al aprendizaje de las ciencias, tales como las ideas previas de los estudiantes, el uso de modelos y abstracciones, la complejidad asociada a la experimentación científica o la carencia de contextos significativos.

Por último, a luz de la discusión anterior, se discuten los posibles efectos de las aplicaciones tecnológicas en la asimilación significativa y duradera de las ideas científicas, así como en el desarrollo de competencias específicas, señalando limitaciones y oportunidades relacionadas con el uso de las TIC.

\section{INFLUENCIA DE LAS TIC EN LA ENSEÑANZA DE LAS CIENCIAS}

Para entender la relevancia y envergadura del impacto de la tecnología en el aprendizaje de las ciencias, sería necesario identificar previamente cuáles son los principales obstáculos que nos encontramos en la enseñanza de las ideas científicas y reflexionar sobre su origen. Este enfoque nos permitirá valorar hasta qué punto las actuales aplicaciones tecnológicas (laboratorios virtuales, simulaciones, herramientas de modelización, de adquisición y representación de datos, de trabajo colaborativo asistido por ordenador...) nos pueden ayudar a afrontar dichos obstáculos y a facilitar el aprendizaje.

De acuerdo con la visión constructivista, los individuos aprenden significativamente cuando son capaces de encontrarle sentido al nuevo conocimiento al conectarlo con lo que ya saben, o integrarlo dentro de sus propios esquemas cognitivos. Desde este punto de vista, el conocimiento preexistente juega un papel clave en la capacidad del sujeto para asimilar nueva información de forma duradera y eficaz.

Por ello, la expansión de las concepciones constructivistas sobre el aprendizaje promovió en las décadas de los ochenta y los noventa un extenso número de investigaciones, destinadas a conocer las ideas previas del alumnado en relación con los contenidos de ciencias. Los resultados de estos trabajos revelaron que muchas de las concepciones de los estudiantes sobre los fenómenos naturales y cotidianos son opuestas a las científicas (Driver et al., 1999). Desde el enfoque constructivista, la incoherencia entre las ideas previas y los conocimientos que se deben enseñar supone un serio obstáculo para la conexión de la nueva información con la preexistente, esto es, para el desarrollo de un aprendizaje significativo. Ser conscientes de la influencia de las ideas previas de los alumnos y reflexionar sobre su origen nos puede permitir comprender mejor el modo en que los individuos desarrollan el conocimiento y diseñar aproximaciones más eficaces para promover el aprendizaje significativo de los conceptos y las teorías científicas.

En la literatura existe un importante número de investigaciones orientadas a facilitar el cambio conceptual en los estudiantes (Hewson et al., 2003), entendido en su sentido original más estricto, como la sustitución de sus concepciones intuitivas por las científicas. Sin embargo, este objetivo no resulta nada fácil, ya que la investigación educativa ha mostrado repetidas veces que las concepciones 
de los estudiantes relacionadas con fenómenos naturales y cotidianos no son caprichosas ni aleatorias, sino que están fuertemente arraigadas en su experiencia personal y en el lenguaje. Además, tienen un importante valor práctico para el individuo, ya que generalmente le permiten desenvolverse satisfactoriamente en situaciones diarias. Por ello, estas ideas previas son muy resistentes al cambio y se mantienen aun después de muchos años de enseñanza formal.

Un creciente número de investigaciones revela que, en el área de ciencias, los estudiantes mantienen múltiples visiones sobre un mismo fenómeno, en lugar de privilegiar las explicaciones de los libros de texto o las sostenidas por los expertos (Linn, 2002). Esto ha promovido una tendencia a hablar de integración de conocimiento o de desarrollo conceptual, en lugar de pretender el cambio conceptual o la erradicación de las preconcepciones del alumnado (Pozo y Gómez-Crespo, 2009).

La existencia en los sujetos de múltiples estructuras explicativas en relación con un mismo fenómeno está también presente en la ciencia. Los científicos mantienen distintas visiones sobre temas como termodinámica, electricidad o mecánica, y recurren a varios niveles de análisis en función del enfoque escogido, del contexto o de las demandas de la situación estudiada. Por ejemplo, cuando se entrevista a físicos y químicos sobre la naturaleza del calor y la temperatura, aplican tanto modelos microscópicos corpusculares, como modelos macroscópicos de flujo de calor (Linn, 2002).

Desde esta perspectiva, el objetivo de la enseñanza de las ciencias, más que sustituir las ideas espontáneas por las científicas, es dotar al individuo de nuevos modelos explicativos para interpretar el mundo y ayudarle a reconocer que el conocimiento científicos es, en muchos casos, más apropiado que sus preconcepciones para describir y/o comprender determinados fenómenos (Gómez-Crespo et al., 2004).

Los modelos y las teorías científicas adquirirán relevancia para los estudiantes si les proporcionamos repetidas oportunidades de comprobar su utilidad y su potencial explicativo. Las prácticas de laboratorio han sido tradicionalmente empleadas en la enseñanza de las ciencias para demostrar las teorías científicas. Bien diseñadas, permiten cuestionar las ideas alternativas de los alumnos formuladas como hipótesis previas a los experimentos, así como encontrar sentido a las ideas científicas cuando son aplicadas para explicar fenómenos.

No obstante, la introducción en las clases de ciencias de experiencias prácticas que favorezcan el cambio o el desarrollo conceptual del alumnado depende, en cierta medida, de los recursos materiales disponibles. El empleo de simulaciones, laboratorios virtuales, visualizaciones o laboratorios remotos ha abierto un nuevo abanico de posibilidades en la búsqueda de contextos significativos para el aprendizaje del conocimiento científico.

\section{Laboratorios virtuales y enseñanza de las ciencias}

Hennessy y colaboradores (2007) han publicado los resultados más relevantes asociados a la evaluación de dos proyectos de investigación sobre enseñanza de las ciencias apoyada en las nuevas tecnologías en el Reino Unido. El trabajo pone de manifiesto una tendencia creciente en los profesores a hacer uso de herramientas tecnológicas para involucrar al alumnado en la formulación de hipótesis y predicciones, que pueden ser posteriormente cuestionadas a través de experimentos virtuales y simulaciones. No obstante, se llama la atención sobre la importancia de dirigir la utilización de dichos instrumentos, de modo que se genere el conflicto cognitivo necesario para poner en crisis las concepciones previas de los estudiantes y reforzar las científicas. Es fundamental tomar conciencia de que, aunque las aplicaciones tecnológicas ofrecen nuevas posibilidades y contextos, la mera utilización del recurso no garantiza la mejora del aprendizaje, es más, en ocasiones, la información ofrecida es erróneamente interpretada por los individuos y puede incluso reforzar sus errores conceptuales. De ahí la importancia de investigar sobre la aplicación de los materiales, de modo que se puedan diseñar y consolidar estrategias y secuencias pedagógicas óptimas, para promover y dirigir el aprendizaje a través de su utilización. 
Sin embargo, al margen de su potencial para cuestionar las ideas de los alumnos y generar conflictos cognitivos, las prácticas de laboratorio han ocupado siempre un lugar destacado en la enseńanza de las ciencias. El papel relevante de las prácticas de laboratorio se puede justificar asumiendo que ayudan a entender la influencia de la experimentación en el desarrollo de conocimiento científico. Además, promueven en los estudiantes interesantes actitudes y habilidades tales como la capacidad de formular problemas e hipótesis, de diseñar experiencias para comprobarlas, de abordar la observación sistemática, la adquisición, representación y análisis de datos, la interpretación crítica de resultados, etc.

Las nuevas posibilidades ofrecidas por la tecnología han llevado a un número importante de autores a reconsiderar las prácticas de laboratorio intentando aprovechar el potencial de simulaciones, visualizaciones y otros recursos didácticos digitales, para favorecer el aprendizaje y optimizar el tiempo presencial disponible en el laboratorio.

En este sentido, algunos investigadores (Quesada et al., 2009) han desarrollado proyectos de innovación enfocados al diseño de material digital multimedia para las prácticas de ciencias experimentales. Estos autores justifican su iniciativa argumentando que, frecuentemente, los alumnos llegan al laboratorio con muy poca o ninguna experiencia previa, por lo que en su mayoría carecen del conocimiento y las habilidades necesarias para manejar el instrumental específico y desarrollar satisfactoriamente las tareas requeridas. Estas circunstancias hacen de las prácticas de laboratorio una estrategia pedagógica poco eficaz, ya que corren el riesgo de quedar reducidas al seguimiento de un protocolo o receta, o a meras tareas mecánicas con escaso valor para promover el conocimiento. Debido a que los estudiantes carecen inicialmente de formación especializada, es necesario invertir tiempo y esfuerzo en familiarizarlos con el manejo y uso adecuado del instrumental específico, lo que repercute de forma negativa en el tiempo disponible para abordar otro tipo de objetivos didácticos.

Teniendo en cuenta las consideraciones anteriores, se propone el diseño de material digital multimedia (vídeos, tutoriales, bancos de preguntas de evaluación), para complementar las prácticas de laboratorio. El material generado está concebido para ser utilizado como actividades pre y postlaboratorio enfocadas a potenciar el aprendizaje y optimizar el tiempo disponible en el laboratorio. Además, los materiales digitales generados pueden ser utilizados por el alumnado de forma autónoma y flexible, adaptándose a cada estilo o ritmo personal. Por ejemplo, antes de las sesiones presenciales, los alumnos pueden disponer de demostraciones sobre cómo manejar el instrumental especializado o cómo llevar a cabo de forma correcta operaciones básicas. Estos recursos están destinados a ayudarlos a familiarizarse con estos aspectos antes de realizar las prácticas. A su vez, los tests de pre-autoevaluación les permiten activar conocimientos y focalizar su atención en los aspectos clave. Tras la utilización del recurso o el desarrollo de la práctica en el laboratorio, los tests de post-autoevaluación hacen posible comprobar el nivel de comprensión de los conceptos o ideas fundamentales, o el grado de dominio de los procedimientos y habilidades puestos en juego.

\section{Herramientas de adquisición y representación de datos}

Tras mencionar el papel de la experimentación en la enseñanza de las ciencias y comentar brevemente cómo la tecnología está promoviendo un cambio en la forma de abordar las prácticas de laboratorio, es apropiado hablar de una actividad directamente relacionada con la experimentación científica: la adquisición de datos. Hoy en día, existen numerosas herramientas tecnológicas que han permitido agilizar los procesos de adquisición de información, automatizando tediosas rutinas mecánicas de cuestionable valor didáctico. Del mismo modo, se han desarrollado aplicaciones para la gestión y representación de datos que facilitan tanto el almacenamiento, como el procesamiento de estos y la búsqueda de interrelaciones entre ellos. Desde hace ya bastantes años, algunos especialistas se han cuestionado el potencial didáctico de estas herramientas para la enseñanza de las ciencias. La adquisición e inter- 
pretación de datos son tareas básicas dentro de las metodologías científicas y, por tanto, familiarizar a los estudiantes con estos procesos no solo les ayuda a entender la forma en que la ciencia desarrolla conocimiento, sino también les permite encontrar sentido a muchas de las teorías científicas cuando ven que estas permiten describir y explicar muchos fenómenos naturales y cotidianos.

El proyecto «El ordenador como compañero de aprendizaje» es un ejemplo interesante sobre cómo se pueden utilizar herramientas de adquisición y representación de datos para la enseñanza de las ciencias (Linn, 2003b). El proyecto gira en torno al diseño de una propuesta didáctica para el estudio de contenidos de termodinámica a lo largo de 12 semanas. En él se recurre a la aplicación de un instrumento de detección y colección de datos en tiempo real. La forma de orientar y guiar a los alumnos en la construcción de conocimiento a través de esta herramienta ha sido refinada gracias a ocho ciclos iterativos de implementación, evaluación y posterior modificación. La versión final del proyecto muestra una mejora del $400 \%$ en la distinción entre calor y temperatura y en la comprensión de los conceptos clave por parte de los estudiantes (Linn, 2003b).

Los investigadores implicados en este proyecto defienden la influencia positiva de lo que ellos denominan «hacer visible el pensamiento». En efecto, a veces las dificultades de aprendizaje específicas asociadas a las ciencias de la naturaleza residen, en parte, en el carácter abstracto de algunas de las ideas y teorías científicas. En este ejemplo, se muestra cómo a través de la adquisición y representación de datos sobre calentamiento y enfriamiento de objetos, los fenómenos de transmisión de calor y de variación de la temperatura se pueden hacer «más visibles» para los estudiantes, facilitando de esta manera la asimilación conceptual y promoviendo un aprendizaje más eficaz y significativo.

No obstante, numerosas experiencias con el alumnado muestran que este, en general, no está familiarizado con las formas de representación de datos y encuentra dificultad para interpretar gráficos. Además, en muchas ocasiones, no es fácil llegar a las ideas científicas a partir de datos experimentales, ya que para ello no solo se requiere un adiestramiento en los lenguajes y formas de representación específicas, sino también la posesión de conocimiento disciplinar sobre la materia (Linn, 2003a). Por ello, si queremos conseguir una aplicación eficaz de estas herramientas tecnológicas para la enseñanza de las ciencias, hemos de diseñar y evaluar cuidadosamente las propuestas didácticas. Estas propuestas deben partir del conocimiento y las habilidades previas de los individuos y ser capaces de guiarlos adecuadamente para encontrar sentido a las ideas científicas a partir de los datos representados.

\section{Entornos virtuales, modelización y enseñanza de las ciencias}

Continuando nuestro análisis sobre el potencial de las actuales aplicaciones tecnológicas para la enseñanza de las ciencias, no podemos pasar por alto las posibilidades asociadas a las herramientas de modelización. De nuevo, el análisis de dichas posibilidades se lleva a cabo desde el punto de vista de la naturaleza del conocimiento cuyo aprendizaje se pretende promover, de las dificultades específicas asociadas con dicho aprendizaje y de los objetivos formativos que nos planteamos como profesionales de la didáctica de las ciencias experimentales.

Algunos autores defienden una visión de la ciencia como una actividad humana en la que la construcción de modelos para explicar y predecir fenómenos juega un papel fundamental (Justi, 2006). Por ello, la comprensión de qué es un modelo y, por lo tanto, la contribución de la modelización al desarrollo del conocimiento científico, debe ser uno de los objetivos de la enseñanza de las ciencias.

Desde este punto de vista, el docente ha de ser consciente de que el término modelo lleva asociados múltiples significados en la experiencia cotidiana del alumnado y, por lo tanto, el empleo de este concepto en el contexto de la enseñanza de las ciencias ha de ser debatido, de modo que se garantice la construcción de nuevos significados en torno a la comprensión de qué es un modelo científico. 
De acuerdo con Justi (2006), en el desarrollo y la aplicación del conocimiento científico, independientemente de la forma de representación empleada, los modelos son representaciones mentales mediante las cuales los científicos razonan (Giere, 1999; Gilbert, 1993; Nersessian, 1999). Los modelos, por tanto, se pueden definir como representaciones del mundo producidas por el pensamiento humano (Giere, 1999), a las cuales se atribuyen distintas funciones: simplificar fenómenos complejos, ayudar en la visualización de entidades abstractas, servir de apoyo en la interpretación de resultados experimentales, ayudar en la elaboración de explicaciones y permitir realizar previsiones sobre los fenómenos modelizados (Justi, 2006).

Los modelos se pueden considerar representaciones mediadoras entre la realidad y la teoría. Aunque un modelo se asocia a una representación concreta de entidades, hechos o fenómenos, el proceso de construcción de modelos implica una actividad mental intensa, en la que se establecen relaciones e interconexiones entre conceptos. Por ello, la importancia de los modelos en la enseńanza de las ciencias no solo se justifica atendiendo al papel que estos juegan en el desarrollo de conocimiento científico, sino también está asociada a su potencial para promover los procesos cognitivos en el estudiante y el desarrollo de un aprendizaje significativo. Otro aspecto positivo asociado al uso de las herramientas de modelización es que la visualización gráfica de la relación entre los distintos factores implicados en un fenómeno facilita el aprendizaje, al hacer más visible el pensamiento y las formulaciones abstractas.

Algunos autores (Alessi y Trollip, 2001) definen una simulación educativa como un modelo de un fenómeno o actividad que los usuarios, en este caso los estudiantes, pueden aprender a través de la interacción con la simulación, de ahí que no podamos hablar de la importancia de la modelización en la enseñanza de las ciencias sin mencionar las posibilidades que ofrecen las simulaciones. Una simulación puede considerarse, por tanto, un programa de ordenador que permite al usuario interactuar con la representación informática de un modelo del mundo natural, de la física o bien de un sistema teórico.

Dentro del ámbito nacional, el Ministerio de Educación de España pone a disposición de los docentes numerosas simulaciones asociadas al proyecto Newton (Segura, 2006). Uno de los principales objetivos de este proyecto es animar al profesorado a integrar dichas simulaciones en su práctica diaria, elaborando secuencias didácticas adecuadas para adaptarlas a su alumnado. También se pretende que el docente emita una valoración del uso de la aplicación tecnológica. Aunque el programa lleva en funcionamiento varios años, no son tantos los profesores que se animan a participar. Las experiencias desarrolladas se publican anualmente en la web específica del proyecto $(<\mathrm{http}$ ://recursostic.educacion. es/eda/web/newton/newton_inicio.html>). Dentro de las conclusiones alcanzadas, encontramos tanto aspectos positivos como negativos, lo que pone de manifiesto que no es el recurso en sí lo que garantiza una mejora en el aprendizaje, sino el criterio pedagógico que guía su utilización. Por ello, tal y como se mencionará más adelante, la integración de simulaciones y otras herramientas tecnológicas en la enseñanza de las ciencias ha de ser orientada por la investigación educativa y frecuentemente requiere sucesivos ciclos de aplicación, evaluación, refinamiento y mejora. A continuación vamos a describir algunas iniciativas que creemos pueden ser consideradas referentes de interés.

Varios grupos de investigación han estudiado y puesto a punto entornos virtuales, recursos digitales y propuestas didácticas en las que se recurre a la construcción y análisis de modelos para promover el aprendizaje en los estudiantes.

Un ejemplo interesante que ilustra las valiosas posibilidades de las herramientas de modelización para la enseñanza de las ciencias se puede encontrar en el proyecto Co Lab (van Joolingen et al., 2005). Este proyecto europeo se ha centrado en el desarrollo y la evaluación de un entorno virtual para promover el aprendizaje de las ciencias de la naturaleza en los niveles superiores de enseńanza secundaria y en la universidad, a través de la realización de proyectos de investigación de forma colaborativa. El trabajo está fundamentado en una concepción del aprendizaje que defiende que los individuos construyen conocimiento de forma significativa, cuando reflexionan y trabajan colaborativamente para 
resolver problemas asociados a contextos y situaciones auténticas. En este sentido, Edelson (2003) argumenta que la gran influencia de los contextos formativos en el aprendizaje se puede justificar aceptando que el conocimiento y las habilidades adquiridas están fuertemente ligados a las situaciones en las que fueron aprendidos. De acuerdo con esta perspectiva, es fundamental tratar de proporcionar actividades y contextos de aprendizaje auténticos, en los que el individuo pueda encontrar sentido y aplicación al nuevo conocimiento (Ariza et al., 2008). En esta línea, se encuentran también las concepciones que entienden el aprendizaje como un proceso de integración del aprendiz en una comunidad de expertos, a través de la práctica y la aplicación del conocimiento.

Desde este marco teórico, se considera que el aprendizaje eficaz de las ciencias exige implicar a los estudiantes en actividades genuinamente científicas, esto es, actividades de experimentación y construcción de modelos. Los experimentos ayudan a comprender las interrelaciones entre los distintos factores involucrados en un fenómeno natural, y la construcción de modelos refleja nuestra comprensión de dichos fenómenos. Mediante esta metodología, no solo se fomenta que los estudiantes encuentren sentido a las ideas científicas para explicar el mundo, sino también que se familiaricen con los procesos de la ciencia.

Co Lab permite a los alumnos trabajar sobre cuatro bloques temáticos relacionados con las ciencias de la naturaleza: la gestión y calidad del agua, el efecto invernadero, mecánica y electricidad.

El entorno virtual de aprendizaje está diseñado metafóricamente, de modo que cada bloque de contenidos queda representado por un edificio. Cada edificio, a su vez, contiene distintas plantas, que simbolizan diferentes niveles de complejidad. Por ejemplo, el bloque sobre tratamiento y calidad del agua incluye un nivel inicial donde se utiliza una simulación de un tanque de agua para realizar experimentos. El nivel inmediatamente superior se basa en el acceso remoto a un tanque real de agua y el último nivel permite trabajar desde una perspectiva más compleja, donde se utiliza una simulación en la que se tiene en cuenta la influencia de diversos factores tales como las lluvias, el cauce de los ríos, el sistema de bombas del depósito de agua, etc. El diseño estructural del entorno permite, por tanto, trabajar con poblaciones de estudiantes de distintas edades o responder a diferentes necesidades educativas, y ofrece un proceso progresivo de aprendizaje con un nivel creciente de complejidad.

Otro aspecto interesante del proyecto reside en el tipo de actividades promovidas. Continuando con la metáfora de los edificios, hemos de señalar que cada tipo de tarea de aprendizaje está asociado con una clase de habitación. De este modo, la fase de experimentación se lleva a cabo en la estancia denominada «laboratorio», mientras que la modelización tiene lugar en «la habitación de teoría». Por su parte, la «sala de reuniones» es el espacio donde los miembros de un grupo se ponen en contacto para informarse sobre los objetivos asociados al proyecto de investigación, asignar tareas, planificarse y hacer un seguimiento del trabajo en equipo.

Tal y como se ha mencionado anteriormente, el proyecto Co Lab pretende involucrar a los estudiantes en tareas colaborativas que los investigadores consideran especialmente significativas para promover la comprensión y el aprendizaje eficaz de las ideas y los procesos científicos, esto es, actividades de modelización y experimentación. Para ello, en primer lugar se solicita a los estudiantes que formulen sus hipótesis sobre el fenómeno investigado antes de iniciar su estudio. Sin embargo, uno de los aspectos más originales de este entorno virtual es que la formulación de hipótesis no se hace mediante enunciados verbales, sino recurriendo a una herramienta de modelización para expresar la presunta relación entre las variables implicadas en cada proceso. Posteriormente, en la zona de experimentación, a través de una simulación o de un laboratorio remoto, los alumnos tienen la oportunidad de comprobar si los resultados experimentales obtenidos se ajustan a su modelo inicial, o si por el contrario es necesario corregir o refinar el modelo.

En este caso, la utilización de una herramienta de modelización, tanto para la formulación inicial de hipótesis, como para la representación de los datos experimentales, permite a los alumnos entender 
la naturaleza y el papel de los modelos. Así, se promueve en ellos un concepto de modelo como una manera de representar las interrelaciones entre las variables de un sistema, que permite explicar fenómenos y hacer predicciones.

Además del entorno virtual de aprendizaje Co Lab, existen otras iniciativas interesantes basadas en la aplicación de las nuevas tecnologías para la enseñanza de las ciencias. En este sentido, cabe mencionar el proyecto asociado al diseño y desarrollo del entorno WISE (Web-based Inquiry Science Environment). Se trata de un entorno virtual financiado por la Fundación Nacional para la Ciencia en los Estados Unidos (National Science Foundation), al que se puede acceder libremente a través de la dirección <http://wise.berkeley.edu/>. El principal propósito de este sitio web es ofrecer recursos basados en las nuevas tecnologías que faciliten la implementación de un aprendizaje de las ciencias a través de la realización de proyectos de investigación por parte de los alumnos.

WISE permite a los estudiantes aprender a plantear problemas e hipótesis, buscar información, criticar ideas y puntos de vista tomando como punto de partida la interpretación de experimentos y evidencias, construir y debatir argumentos y desarrollar y cuestionar modelos. Para ello, el entorno incorpora simulaciones, herramientas de adquisición de datos, de modelización, visualizaciones y diversas páginas y vínculos que aportan información relevante sobre la que reflexionar, discutir y revisar ideas. Estas actividades son abordadas en el transcurso de un proyecto de investigación sobre algún tópico de interés (cambio climático, reciclado, coches híbridos, alimentos transgénicos...). Todo lo que los estudiantes necesitan para llevar a cabo uno de los proyectos incluidos en el entorno virtual WISE es un ordenador con navegador y conexión a Internet. Los proyectos están diseñados para desarrollarse a lo largo de una semana de curso académico, siendo integrados en el resto del currículo.

Uno de los aspectos más interesantes de WISE es que invita a cualquier profesional interesado en la generación de recursos para la enseñanza de la ciencia (profesores, investigadores, diseñadores, tecnólogos...) a añadir nuevos proyectos de investigación a su base de datos, o hacer modificaciones o propuestas de mejora sobre los ya existentes. Con este propósito, se ofrecen herramientas de autor y patrones de diseño basados en la investigación educativa, los cuales incluyen todos aquellos elementos que juegan un papel clave en la promoción de un aprendizaje eficaz y que facilitan la integración de conocimiento.

Cada vez que se envía una nueva propuesta de proyecto de investigación, el equipo de profesionales involucrados en el diseño de los recursos educativos establece un plan de evaluación de dicho proyecto. Normalmente, se recurre a su implementación con un grupo de alumnos. La evaluación del potencial pedagógico del proyecto se basa en los resultados de su aplicación en el aula y el análisis de la actuación de los participantes. Los proyectos se refinan a través de ciclos sucesivos de implementación, evaluación y mejora. Aquellos proyectos que demuestran un impacto significativo en el aprendizaje de los estudiantes y en su comprensión de las ideas científicas son incorporados a la librería WISE. Esta base de datos incorpora más de veinticinco proyectos en inglés y algunos pocos en otras lenguas (alemán, holandés, noruego...).

La iniciativa descrita pretende favorecer el enriquecimiento derivado de la colaboración entre los profesionales de la enseñanza de las ciencias y permitir que los distintos colectivos (profesores, estudiantes, académicos, diseñadores...) se beneficien de los resultados de la investigación educativa asociada a la evaluación de las innovaciones pedagógicas.

\section{El diseño de enseñanza basado en investigación: aplicación a las innovaciones didácticas basadas en las TIC}

La metodología aplicada por el proyecto WISE para el diseño, evaluación y mejora de los recursos educativos generados se puede incluir dentro del paradigma conocido en la literatura internacional como 
«Design-Based Research» o diseño de enseñanza informado por los resultados de la investigación. El principal propósito de esta aproximación es favorecer la conexión entre la teoría y la evaluación del impacto de los nuevos recursos didácticos mediante su implementación en contextos reales. Con ello se persigue no solo poner la investigación educativa al servicio de la mejora del aprendizaje, sino que la evaluación de la innovación pedagógica haga posible identificar los rasgos y patrones de diseño que promueven un aprendizaje eficaz. De este modo, la implementación práctica permite la elaboración de principios teóricos de diseño basados en la investigación educativa.

Algunos autores han adoptado este paradigma para la evaluación de recursos didácticos apoyados en las nuevas tecnologías. Por ejemplo, Hoadley (2002) utilizó esta metodología para mejorar el diseño de entornos de aprendizaje virtual y discusiones on-line.

Otro ejemplo de la aplicación del diseño de enseñanza informado por los resultados de investigación puede encontrarse en el artículo publicado por Sandoval y Reiser (2004). El trabajo describe el proceso de desarrollo de recursos digitales integrados en un entorno para el aprendizaje de las ciencias a través de la indagación. La valoración de las herramientas tecnológicas a través de sucesivos ciclos de implementación, evaluación y revisión mostró deficiencias y permitió identificar los aspectos susceptibles de mejora. Uno de estos elementos que se quería mejorar estaba relacionado con la necesidad de aportar un mayor grado de orientación sobre la actividad investigadora y reflexiva de los alumnos.

\section{Enseñanza de las ciencias, herramientas de discusión y trabajo colaborativo}

El debate y la discusión han sido ampliamente reconocidos como estrategias pedagógicas adecuadas para promover la reflexión y el cambio conceptual. En este sentido, el trabajo colaborativo ofrece oportunidades valiosas para promover la construcción social del conocimiento a través de la comunicación y el intercambio de ideas entre los miembros del grupo. Muchos autores defienden que los individuos construyen significado mediante el uso del lenguaje y la interacción con otros y, por ello, el trabajo colaborativo ofrece un contexto ideal para ejercer la reflexión, la revisión de ideas y el establecimiento de relaciones conceptuales, esto es, el aprendizaje significativo (Gibbings y Brodie, 2008; Kearney, 2004; Moreno et al., 2007).

De acuerdo con Ariza y colaboradores (2008), cuando se diseñan tareas de trabajo colaborativo en las que se requiere que cada participante explique y defienda sus puntos de vista, los estudiantes han de meditar sobre sus experiencias previas y han de buscar razones y argumentos para justificar sus ideas y expresarlas de forma clara y convincente. Los procesos de fundamentación de ideas y reflexión permiten contrastar las creencias y concepciones personales con las de otros, o revisar su coherencia con las evidencias disponibles, cuestionando su adecuación para explicar fenómenos. Todos estos procesos cognitivos fomentan el cambio conceptual y están asociados al desarrollo de un aprendizaje significativo.

Sin embargo, el diálogo y el debate han sido actividades ampliamente utilizadas en la enseńanza y en la construcción de conocimiento y fueron empleadas ya en la Grecia antigua, por lo que en sí mismas no suponen ninguna innovación didáctica. Pero ¿existe alguna diferencia significativa cuando estas actividades se realizan a través de las tecnologías de la información y la comunicación? Si asociamos el trabajo en equipo con la oportunidad de promover la construcción social del conocimiento, ‘aporta el trabajo colaborativo a través del ordenador alguna ventaja respecto al trabajo en equipo tradicional?

Una respuesta evidente que acude rápidamente a la mente de cualquiera podría ser la mayor flexibilidad temporal y espacial ofrecida por las TIC. Los chats, videoconferencias, correos electrónicos, foros virtuales y otras herramientas tecnológicas colaborativas permiten la comunicación y el debate entre personas situadas en lugares remotos y en diversas localizaciones geográficas. Tampoco se requiere que los participantes se encuentren presentes al mismo tiempo, ya que las herramientas de comunicación asíncrona ofrecen un espacio donde quedan recogidas las distintas aportaciones a lo largo del tiempo. 
La investigación educativa parece indicar que el trabajo colaborativo apoyado en las TIC ofrece otras ventajas adicionales. Por ejemplo, Linn $(2003 a)$ aporta evidencias de que, en promedio, solo un $10 \%$ de la clase suele participar en los debates tradicionales en el aula, mientras que cuando se recurre a actividades adecuadamente diseñadas para promover la discusión a través de foros virtuales, se consigue la participación de más del $90 \%$ de la clase. Además, se ha demostrado que la calidad de las aportaciones hechas por los estudiantes también se ve afectada de algún modo. Los alumnos ofrecen más explicaciones y justificaciones para fundamentar sus afirmaciones y prestan más atención a los argumentos esgrimidos cuando saben que estas están destinadas a permanecer escritas en el foro y a ser leídas y evaluadas por sus compañeros. Linn (2003a) también señala que los debates a través de foros electrónicos ofrecen a los estudiantes mayores oportunidades para reflexionar, ya que les permiten pensar antes de escribir. Por el contrario, el flujo continuo de las discusiones en clase favorece la participación de los más impulsivos y extrovertidos y a menudo hace que los más reflexivos queden callados, tomándose su tiempo para meditar. Otra ventaja de las herramientas digitales respecto al debate presencial es que las primeras permiten al docente o al investigador disponer del registro de las contribuciones de cada individuo y aportan información para hacer un seguimiento del comportamiento y participación de cada estudiante.

Sin embargo, las herramientas tecnológicas que facilitan el trabajo colaborativo y la discusión no garantizan la reflexión de los alumnos, ni mucho menos, la construcción social del conocimiento. Más aún, un debate puede llegar a ser tedioso, frustrante o incluso perjudicial para la comprensión de los conceptos científicos cuando no se proporciona la orientación necesaria para reconducir las ideas erróneas. Por ello, los docentes juegan un papel clave en el diseño, seguimiento y retroalimentación asociados a una actividad de discusión. El análisis de entrevistas destinadas a profundizar en la opinión de los alumnos participantes en foros electrónicos muestra que más de un $54 \%$ conceden especial importancia a la intervención y orientación del docente y reclaman un mayor grado de retroalimentación (Puntambekar, 2006).

La literatura especializada pone de manifiesto los efectos beneficiosos asociados a las actividades de aprendizaje colaborativo cuando estas han sido cuidadosamente diseñadas (Johnson y Johnson, 1999). Scardamalia y Bereiter (1992) llevaron a cabo una serie de estudios que contribuyeron a identificar qué características del diseño de este tipo de actividades favorecían la construcción de conocimiento. Algunas de las conclusiones derivadas de estos estudios apuntan a la necesidad de seleccionar cuidadosamente los temas de discusión, aportar información relevante y evidencias que lleven a los alumnos a cuestionar ideas erróneas o a reconocer la utilidad de las concepciones científicas y proporcionar directrices adecuadas para reconducir la reflexión y favorecer la participación.

\section{CONSIDERACIONES FINALES}

El presente artículo pretende fomentar la reflexión en torno al uso de las TIC en la enseñanza de las ciencias y a su potencial para facilitar el aprendizaje y promover un conocimiento duradero y transferible. Con este propósito, se han descrito investigaciones basadas en la aplicación didáctica de herramientas de adquisición de datos, de programas de modelización, de laboratorios virtuales y de herramientas de discusión y trabajo colaborativo. Así mismo, se debate sobre la utilización de las nuevas tecnologías en el diseńo de actividades de pre y postlaboratorio. La literatura especializada muestra que el empleo de recursos digitales multimedia en actividades de pre y postlaboratorio permite aumentar el valor formativo de las prácticas de ciencias experimentales y optimizar el tiempo disponible en las sesiones presenciales. 
Sin embargo, con este trabajo también se persigue aportar una visión crítica que nos permita tomar consciencia de que no es el medio en sí utilizado para la enseñanza, sino el uso pedagógico que se haga de él, lo que provoca una diferencia significativa en el aprendizaje adquirido. Para ello, se discuten algunos resultados de la investigación especializada. Por ejemplo, la experiencia muestra que los alumnos no siempre interpretan de forma adecuada los gráficos e imágenes ofrecidos por las visualizaciones y los programas de modelización. Por lo tanto, si no se les adiestra en las formas de representación específicas, o si carecen de la orientación oportuna, los recursos digitales pueden reforzar sus errores conceptuales, en lugar de facilitar la asimilación y comprensión de las ideas científicas. Más aún, aunque las herramientas de adquisición de datos en tiempo real y los laboratorios virtuales ofrezcan nuevas oportunidades para encontrar sentido y aplicabilidad a las teorías científicas, no siempre es fácil inferir estas a partir de un conjunto de datos experimentales, por lo que se requiere la intervención del docente para guiar al alumno en la construcción de conocimiento.

El artículo presenta también un análisis sobre el valor del debate y la reflexión para promover el cambio y el desarrollo conceptual del alumno, así como la asimilación significativa de las ideas científicas para explicar el mundo. En esta línea, se revisan investigaciones sobre trabajo colaborativo asistido por ordenador y la influencia de los foros virtuales en la construcción social del conocimiento, señalando ventajas y riesgos. Continuando con nuestro análisis crítico, se llama la atención sobre la importancia de diseñar adecuadamente las experiencias colaborativas apoyadas en las nuevas tecnologías y la pertinencia de aportar el nivel adecuado de orientación y retroalimentación en las discusiones a través de foros digitales.

Algunos autores muestran que no todos los recursos tecnológicos son igual de potentes para promover el aprendizaje y que incluso cuando nos centramos en las aplicaciones más exitosas, existen múltiples factores que condicionan los resultados de su implementación en el aula (Roschelle et al., 2000). Por ello, sería conveniente recurrir a los resultados de la investigación que tratan de contribuir al uso eficaz de simulaciones y otros recursos digitales (Blake y Scanlon, 2007; Hennessy et al., 2007; Krange y Ludvigsen, 2009; Wilensky, 2003).

Las investigaciones sobre la aplicación de la tecnología a la enseñanza de las ciencias ponen de manifiesto la necesidad de ir refinando y mejorando los entornos virtuales y los recursos digitales generados, a través de ciclos sucesivos de implementación, evaluación y revisión. En este sentido, consideramos que el diseño pedagógico informado por los resultados de la investigación educativa (Design Based Research) no solo permite la mejora continua de la práctica docente, sino que constituye un paradigma adecuado para la evaluación de las innovaciones pedagógicas basadas en las TIC.

El análisis del impacto de las TIC sobre la enseńanza de las ciencias se aborda en todo momento desde una visión especializada, basada en el conocimiento actual acerca de cómo los individuos aprenden ciencias. Se ha prestado especial atención a los principales obstáculos asociados a la comprensión de las ideas científicas (ideas previas de los alumnos, abstracción de los modelos y teorías, carencia de contextos significativos...). Desde esta perspectiva y a pesar de las limitaciones y los riesgos descritos anteriormente, consideramos que las TIC ofrecen recursos muy potentes para facilitar y potenciar el aprendizaje de las ciencias. Por un lado, estas herramientas facilitan el acceso a la información y multiplican las posibilidades de interacción y comunicación, superando barreras temporales y espaciales. Por otro lado, las simulaciones, las modelizaciones y los laboratorios virtuales aumentan las oportunidades de cuestionar concepciones erróneas y acceder a contextos significativos para la comprensión y aplicación de las teorías científicas. Además, ponen a disposición del docente la eficacia de las imágenes para representar y hacer mucho más visibles los modelos y teorías abstractas de la ciencia, facilitando su asimilación y permitiendo la conexión entre el ámbito macroscópico o físico y el microscópico o teórico.

La eficacia de la tecnología para potenciar el aprendizaje de diversos contenidos de ciencias queda avalada por numerosas investigaciones. Este es el caso de trabajos sobre la mejora de habilidades 
relacionadas con la resolución de problemas cuantitativos (Diederen et al., 2005), conceptos y procedimientos asociados a la medida de variables físicas (Kiboss, 2002), desarrollo de destrezas espaciales relacionadas con la geografía o la astronomía (Piburn et al., 2005; Hasen et al., 2004) o mejora de la comprensión de conceptos claves en química y física (Trindade et al., 2002; Venkataraman, 2009; Zucker y Hug, 2008).

Fundamentándonos en la perspectiva ofrecida en este trabajo, consideramos que sería interesante facilitar y promover el uso de los actuales recursos y herramientas tecnológicas como medio para afrontar dos de las grandes inquietudes de los profesionales de la enseñanza de las ciencias: motivar a los alumnos hacia la ciencia y ayudarlos a comprender el valor del conocimiento científico para explicar el mundo.

Finalmente y haciéndonos eco de las palabras de Linn publicadas en esta misma revista (2002), nos gustaría hacer un llamamiento a la cooperación entre especialistas, para identificar maneras efectivas de usar y adaptar materiales de enseñanza, a fin de sacar partido de la tecnología en la educación. Creemos que iniciativas de acceso libre a recursos digitales tales como la vinculada al entorno WISE constituyen un vehículo muy valioso para la colaboración, la investigación y la mejora.

\section{BIBLIOGRAFÍA}

Alessi, S. M. y Trollip, S. R. (2001). Multimedia for Learning: Methods and Development (3rd ed.). Boston: Allyn \& Bacon.

Ariza, M. R., Quesada, A., Quijano, R. y Ocaña, M. T. (2008). An experience about promoting general and specific competences acquisition in High Education: autonomous work and collaborative skills in the development of a project aimed at eliciting motivation and contextualized learning. In L. Gómez, D. Belenguer, I. Candel (eds.), International Conference of Education, Research and Innovation (pp. 1-7). Madrid: IATED.

Blake, C. y Scanlon, E. (2007). Reconsidering simulations in science education at a distance: Features of effective use. Journal of Computer Assisted Learning, 23, pp. 491-502. http://dx.doi.org/10.1111/j.1365-2729.2007.00239.x

Diederen, J., Gruppen, H., Hartog, R. y Voragen, A. G. J. (2005). Design and evaluation of digital learning material to support acquisition of quantitative problem-solving skills within food chemistry. Journal of Science Education and Technology, 14(5-6), pp. 495-507. http://dx.doi.org/10.1007/s10956-005-0224-0

Driver, R., Guesne, E. y Tiberghien, A. (1999). Ideas cientificas en la infancia y la adolescencia (4a Edición). Madrid: Morata.

Edelson, D. C. (2003). Realising authentic science learning through the adaptation of scientific practice. In B.J. Fraser y K.G. Tobin (eds.). International Handbook of Science Education, (2a edición, pp. 317-331). London: Kluwer Academic Publishers.

Gibbings, P. y Brodie, L. (2008). Team-based learning communities in virtual space. International Journal of Engineering Education, 24(6), pp. 1119-1129.

Giere, R. N. (1999). Using Models to Represent Reality, en Magnani, L., Nersessian, N. J. y Thagard, P. (eds.). Model-Based Reasoning in Scientific Discovery, Nueva York: Kluwer and Plenum Publishers, pp. 41-57.

Gilbert, J. K. (1993). Models y Modelling in science education. Hatfield: The Association for Science Education. 
Gómez Crespo, M. A., Pozo, J. I. y Gutiérrez, M. S. (2004). Enseñando a comprender la naturaleza de la materia: el diálogo entre la química y nuestros sentidos. Educación Química, 15(3), pp. 198-209.

Hansen, J. A., Barnett, M., Makinster, J. G. y Keating, T. (2004). The impact of three-dimensional computational modelling on student understanding of astronomical concepts: A quantitative analysis. International Journal of Science Education, 26(11), pp. 1365-1378.

http://dx.doi.org/10.1080/09500690420001673766

Hennessy, S., Wishart, J., Whitelock, D., Deaney, R., Brawn, R., Velle, L., Mcfarlane, A., Ruthven, K. y Winterbottom, M. (2007). Pedagogical approaches for technology-integrated Science teaching. Computers and Education, 48(1), pp. 137-152.

http://dx.doi.org/10.1016/j.compedu.2006.02.004

Hewson, P. W., Beeth, M. E. y Thorley, N. R. (2003). Teaching for conceptual change. In B. J. Fraser y K. G. Tobin (eds.). International Handbook of Science Education (2. a edición, pp. 119-218). London: Kluwer Academic Publishers.

Hoadley, C. (2002). Creating context: Design-based research in creating and understanding CSCL, en G. Stahl (ed.). Computer Support for Collaborative Learning Mahwah. NJ: Lawrence Erlbaum Associates, pp. 453-462.

Johnson, D. W. y Johnson, R. T. (1999). Making cooperative learning work. Theory into Practice, 38, pp. 67-73.

http://dx.doi.org/10.1080/00405849909543834

Justi, R. (2006). La enseñanza de ciencias basada en la elaboración de modelos. Enseñanza de las Ciencias, 24(2), pp. 173-184.

Kearney, M. (2004). Classroom use of multimedia-supported predict-observe-explain tasks in a social constructivist learning environment. Research in Science Education, 34(4), pp. 427-453. http://dx.doi.org/10.1007/s11165-004-8795-y

Kiboss, J. K. (2002). Impact of a computer-based physics instruction program on pupils' understanding of measurement concepts and methods associated with school science. Journal of Science Education and Technology, 11(2), pp. 193-198. http://dx.doi.org/10.1023/A:1014673615275

Krange, I. y Ludvigsen, S. (2009). Original article: The historical and situated nature of design experiments - Implications for data analysis. Journal of Computer Assisted Learning, 25, pp. 268-279. http://dx.doi.org/10.1111/j.1365-2729.2008.00307.x

Linn, M. (2002). Promover la educación científica a través de las tecnologías de la información y comunicación (TIC). Enseñanza de las Ciencias, 20(3), pp. 347-355.

Linn, M. (2003a). Technology and science education: starting points, research programs, and trends. International Journal of Science Education, 25(6), pp. 727-758. http://dx.doi.org/10.1080/09500690305017

Linn, M. (2003b). The impact of technology on science instruction: historical trends and current opportunities, en B. J. Fraser y K. G. Tobin (eds.). International Handbook of Science Education (2a edición, pp. 265-294). London: kluwer Academic Publishers.

Linn, M., Clarck, D. y Slotta, J. D. (2003). WISE design for knowledge integration. Science Education, 87(4), pp. 517-538.

http://dx.doi.org/10.1002/sce.10086

Moreno, L., Gonzalez, C., Castilla, I., Gonzalez, E. y Sigut, J. (2007). Applying a constructivist and collaborative methodological approach in engineering education. Computers and Education, 49, pp. 891-915.

http://dx.doi.org/10.1016/j.compedu.2005.12.004 
Nersessian, N. J. (1999). Model-Based Reasoning in Conceptual Change, en Magnani, L., Nersessian, N. J. y Thagard, P. (eds.). Model-Based Reasoning in Scientific Discovery. Nueva York: Kluwer and Plenum Publishers, pp. 5-22.

Piburn, M. D., Reynolds, S. J., Mcauliffe, C., Leedy, D. E. y Johnson, J. K. (2005). The role of visualization in learning from computer-based images. International Journal of Science Education, 27(5), pp. 513-527.

http://dx.doi.org/10.1080/09500690412331314478

Pozo, J. I. y Gómez-Crespo, M. A. (2009). Del conocimiento cotidiano al conocimiento científico: más allá del cambio conceptual, en J. I. Pozo y M. A. Gómez-Crespo (eds.). Aprender y enseñar ciencia. Madrid: Morata, pp. 128-146.

Puntambekar, S. (2006). Analyzing collaborative interactions: divergence, shared understanding and construction of knowledge. Computers and Education, 47(3), pp. 332-351. http://dx.doi.org/10.1016/j.compedu.2004.10.012

Quesada, A., Ariza, M. R., Ortiz, A. y Bermejo, R. (2009). Designing and applying multimedia material for experimental sciences. Teacher and student assessment of its implementation, en L. Gómez, D. Martí e I. Candel. International Technology, Education and Development Conferences. Valencia, Spain: IATED, pp. 4911-4919.

Roschelle, J. M., Pea, R. D., Hoadley, C. M., Gordin, D. N. y Means, B. M. (2000). Changing how and what children learn in school with computer-based technologies. Future of Children, 10, pp. 76-97.

http://dx.doi.org/10.2307/1602690

SAndoval, W. A. y ReIser, B. J. (2004). Explanation-driven inquiry: Integrating conceptual and epistemic scaffolds for scientific inquiry. Science Education, 88(3), pp. 345-372. http://dx.doi.org/10.1002/sce.10130

Scardamalia, M. y Bereiter, C. (1992). A knowledge building architecture for computer supported learning, en E. De Corte, M. C. Linn, H. Mandl, y L. Verschaffel (eds.). Computer-based learning environments and problem solving. Berlin: Springer-Verlag. http://dx.doi.org/10.1007/978-3-642-77228-3_3

Segura, M. (2006). Contenidos educativos digitales en línea del Centro Nacional de Información y Comunicación Educativa (CNICE). Alambique. Didáctica de las Ciencias Experimentales, 50, pp. 9-18.

Trindade, J., Fiolhais, C. y Almeida, L. (2002). Science learning in virtual environments: A descriptive study. British Journal of Educational Technology, 33(4), pp. 471-488. http://dx.doi.org/10.1111/1467-8535.00283

Van Joolingen, W. R., De Jong, T., Lazonder, A. W., Savelsbergh, E. y Manlove, S. (2005). Co-Lab: Research development of an on-line learning environment for collaborative scientific discovery learning. Computers in Human Behavior, 21(4), pp. 671-688.

http://dx.doi.org/10.1016/j.chb.2004.10.039

Venkataraman, B. (2009). Visualization and interactivity in the teaching of chemistry to science and non-science students. Chemistry Education Research and Practice, 10(1), pp. 62-69. http://dx.doi.org/10.1039/b901462b

Wilensky, U. (2003). Statistical mechanics for secondary school: The gaslab multi-agent modeling toolkit. International Journal of Computers for Mathematical Learning, 8, pp. 1-41. http://dx.doi.org/10.1023/A:1025651502936

Zucker, A. A. y Hug, S. T. (2008). Teaching and learning physics in a 1:1 laptop school. Journal of Science Education and Technology, 17(6), pp. 586-594.

http://dx.doi.org/10.1007/s10956-008-9125-3 


\title{
ICT and meaningful science learning
}

\author{
Marta Romero Ariza \\ mromero@ujaen.es \\ Antonio Quesada Armenteros \\ antquesa@ujaen.es \\ Departamento de Didáctica de las Ciencias \\ Universidad de Jaén
}

This paper intends to promote reflection on ICT's potential to facilitate or enhance science learning. In order to contribute to this purpose, key research works focused on the use of technological resources in science education (data acquisition devices, modelling tools, simulations, virtual laboratories...) are reviewed and discussed. The instructional value of these applications is analysed from the perspective of the specific obstacles for science learning and the current understanding about how individuals learn.

Meaningful learning of scientific knowledge is usually obstructed by students' inadequate pre-conceptions about the natural phenomena, by the lack of significant instructional contexts to promote situated and transferable learning, and by the abstract nature of many scientific theories and models. There is also a necessity to provide students with opportunities to appreciate the value of scientific procedures and the role of modelling and theory construction in the development of scientific knowledge. This paper reviews the use of a wide range of technological resources, with the intention of analyzing how they can address specific learning obstacles and support the acquisition of learning goals in science education.

The first section focuses on the utilization of virtual and remote laboratories. They offer more opportunities for experimentation by facilitating the access to specialized lab equipment. Nevertheless, attention is drawn to the importance of not regarding experiments as mere demonstrations but encouraging previous hypothesis formulation as a way to challenge students' preconceptions. Emphasis should be placed on reasoning and understanding, promoting the cognitive processes related to meaningful learning.

Closely linked to experimentation, data acquisition devices coupled with tools for data handling and graphing make it easier for science teachers to engage students in evidence-based arguments and the use of scientific ideas to explain physical and chemical phenomena. Again, risks are pointed out, since the utilization of specialized modes of representation is not always intuitive enough or straightforwardly understood. There is a need to introduce students to the appropriate interpretation of specialized graphs and other scientific languages in order to avoid misunderstanding or the reinforcement of misconceptions.

Another common obstacle for students in science learning is related to the abstract nature of many theories or scientific models to explain natural phenomena. Technology provides a wide range of digital resources to facilitate students' understanding of scientific theories. For instance, through the use of animations and simulations, it is possible to make individuals visualize abstract scientific models and to connect the symbolic or micro-scale world with macroscale properties and behaviours, promoting meaningful and enhanced learning.

Besides facilitating abstract concept visualizations and supporting visual learners, modelling tools help individuals appreciate the nature and role of models in the development of scientific knowledge.

Modelling has also been used as a means to express students' conceptions or hypotheses about natural phenomena. By doing so, the learners' thinking becomes visible, providing a basis to guide instruction and allow conceptual change.

This paper also comments on the potential of some technological resources to widen or enrich learning settings. Researchers have uncovered a great deal of discouraging evidence showing that students are often unable to meaningfully apply the knowledge which they acquire in school. This inability to apply knowledge in real world settings has been attributed to the situated nature of knowledge, that is, the recognition that the knowledge and skills that learning activities produce are tied to the situation in which they are learned.

Finally, the potential of electronic forums and chats to promote the social construction of knowledge through discussion is also analyzed, turning to research to identify key features and successful designs. The role of teachers as facilitators of students' engagement in reasoning and reflection is stressed.

In summary, this paper shows that there is enough research evidence about the potential of technology to facilitate and enhance science learning. However, attention is drawn to the fact that the mere presence of computers in the classroom does not ensure their effective use. Some computer applications have proved to be more successful than others and many factors influence how well even the most promising applications are implemented. Therefore, in order to fully benefit from technology in education, it is necessary to build on previous research outcomes about effective use and promote teachers' training and professional development in this area. This paper intends to contribute to this goal by reviewing key findings from specialized literature and trying to promote reflection on the opportunities and risks associated with the introduction of technological resources in science education. 
